\title{
Actitud diagnóstica y terapéutica ante el paciente que acude con hemoptisis
}

\author{
R. Cortés Sancho, P. Cossío San Jósé, E. Miñambres Alija, J. M. Rodríguez \\ PASCUAL ${ }^{1}$, M. PUYO GIL ${ }^{2}$ \\ Residente de Medicina de Familia y Comunitaria. 'Especialista en Medicina de \\ Familia y Cardiología. C.S. Laguna de Duero. Valladolid. 'Especialista en Neumolo- \\ gía. Servicio de Neumología. Hospital Universitario del Río Hortega. Valladolid
}

\section{RESUMEN}

La hemoptisis es un sintoma que requiere un estudio exhaustivo ya que puede ser secundaria a una enfermedad grave y/o dar lugar a una hemorragia fatal o incluso a un síndrome asfíctico. Por ello debemos confirmar el diagnóstico en el menor tiempo posible, así como evaluar la gravedad del cuadro, localizar la lesión responsable y tratarla adecuadamente porque la evolución clínica es impredecible.

El objeto de este trabajo es repasar el manejo de esta entidad, así como establecer un modelo que coordine los Servicios de Atención Primaria y Neumología.

Palabras clave: Hemoptisis. Etiología. Diagnóstico. Tratamiento.
Diagnostic and therapeutic attitude in patient with hemoptysis

\section{ABSTRACT}

Hemoptysis is a symptom that needs an exhaustive study because it can be secondary to a critical disease and/or give rise to a fatal hemorrhage or even an asphyxia syndrome. For these reasons we have to evaluate and confirm as soon as possible the importance of the disease in charge, to localize the injury and to decide the best treatment, because the clinical course is umpredictable.

The aim of this paper is review the manegement of this symptom as well as to stablish a pattern that allows to coordinate Primary Health Care and Respiratory Medicine.

Key words: Hemoptysis. Etiology. Diagnosis. Manegement.

\section{INTRODUCCIÓN}

La hemoptisis se define como la expulsión de sangre con la tos de procedencia subglótica ${ }^{1}$. En función del volumen de sangre emitido y de la forma de presentación diremos que la hemoptisis es leve si la cantidad de sangre expectorada y generalmente mezclada con el esputo no supera los 15-20 ml/día; franca o moderada ante la expulsión de sangre pura, con un volumen intermedio entre la hemoptisis leve y la masiva; masiva ante un volumen superior a 600 $\mathrm{ml} / 24-48 \mathrm{~h}$ o ritmo superior a $150-200 \mathrm{ml} / \mathrm{h}$, consti- tuyendo una urgencia médica; y amenazante cuando pone en peligro la vida del paciente como consecuencia de la inundación hemática del árbol tráqueobronquial, más que por las posibles consecuencias propias de toda hemorragia importante.

\section{ETIOLOGÍA}

En torno a un centenar de entidades diferentes pueden manifestarse con hemoptisis. La frecuencia relativa de cada una de ellas es variable en las distin- 
tas publicaciones dependiendo de la población estudiada y de las técnicas diagnósticas empleadas². Bronquiectasias, tuberculosis y carcinoma broncogénico han sido durante los años comprendidos entre 1940 y 1961 las causas más frecuentes de hemoptisis (Tabla I) ${ }^{3}$.

Tal y como se observa en los estudios prospectivos más recientes (Tabla II) las causas más comunes de hemoptisis en la actualidad' son: el carcinoma broncogénico (20-28\%), la bronquitis crónica (18-29\%) y las bronquiectasias (10-20\%). Las enfermedades cardiovasculares (infarto pulmonar, estenosis mitral) son res- ponsables del 5\% de las hemoptisis. Menos del 5\% de los sangrados subglóticos se corresponderán con un grupo heterogéneo de patologías: cuerpo extraño tráqueo-bronquial, diátesis hemorrágica, tratamiento anticoagulante, traumatismo torácico, vasculitis tipo Wegener, lupus eritematoso sistémico, síndrome de Goodpasture. En torno al 3\% de los pacientes con hemoptisis padecerán tuberculosis pulmonar.

No obstante, y a pesar de un amplio estudio del paciente, del 8 al $22 \%$ de los sangrados no tendrán confirmación diagnóstica de la causa, denominándose en estos casos hemoptisis idiopática o criptogenética ${ }^{3}$.

Tabla I

\begin{tabular}{|c|c|c|c|c|c|c|c|c|}
\hline \multicolumn{9}{|c|}{ PORCENTAJES DE LAS PRINCIPALES ETIOLOGÍAS DE HEMOPTISIS (SERIES 1942-1961);,4 } \\
\hline & $\begin{array}{c}1942 \\
\text { Jackson }\end{array}$ & $\begin{array}{l}1946 \\
\text { Heller }\end{array}$ & $\begin{array}{c}1948 \\
\text { Abbott } \\
\text { (Atlanta) }\end{array}$ & $\begin{array}{l}1951 \\
\text { Levitt }\end{array}$ & $\begin{array}{c}1952 \\
\text { Moersch } \\
\text { (Mayo) }\end{array}$ & $\begin{array}{l}1952 \\
\text { Souders } \\
\text { (Boston) }\end{array}$ & $\begin{array}{l}1961 \\
\text { Pursel }\end{array}$ & $\begin{array}{c}\text { Medias } \\
\text { (rangos) }\end{array}$ \\
\hline $\mathrm{n}$ & 436 & 413 & 497 & 717 & 200 & 105 & 105 & 2.473 \\
\hline Neoplasia & 20 & 2 & 21 & 12 & 24 & 3 & 19 & $14,4(2-24)$ \\
\hline Bronquiectasias & $\underline{32}$ & 7 & 21 & 15 & $\underline{27}$ & 37 & 23 & $\underline{23}(7-37)$ \\
\hline Bronquitis & 17 & 15 & 2 & 0 & 9 & 12 & 5 & $8,5(0-17)$ \\
\hline Tuberculosis & - & $\underline{39}$ & $\underline{22}$ & $\underline{47}$ & 6 & 2 & 13 & $21,5(2-47)$ \\
\hline Neumonía/abceso & 18 & 4 & 2 & 6 & 8 & 2 & 8 & $7,7(2-18)$ \\
\hline TEP & 0 & 1 & 6 & 0 & 1 & 0 & 3 & $1(0-3)$ \\
\hline Cardiopatía & 0 & 2 & 0 & 7 & 1 & 9 & 3 & $2,8(0-9)$ \\
\hline Idiopática & 8 & 16 & 4 & 5 & 8 & 18 & 15 & $10,6(4-18)$ \\
\hline
\end{tabular}

Tabla II

PORCENTAJES DE LAS PRINCIPALES ETIOLOGÍAS DE HEMOPTISIS (SERIES 1989-2000),

\begin{tabular}{|c|c|c|c|c|c|c|c|}
\hline & $\begin{array}{c}1989 \\
\text { Johnston } \\
\text { (Kansas) }\end{array}$ & $\begin{array}{c}1991 \\
\text { Santiago } \\
\text { (LA) }\end{array}$ & $\begin{array}{c}1994 \\
\text { McGuiness } \\
\text { (NY) }\end{array}$ & $\begin{array}{c}1995 \\
\text { Plaza } \\
\text { (Barcelona) }\end{array}$ & $\begin{array}{c}1997 \\
\text { Hirsberg } \\
\text { (Israel) }\end{array}$ & $\begin{array}{c}2000 \\
\text { Haro } \\
\text { (Albacete) }\end{array}$ & $\begin{array}{l}\text { Medias } \\
\text { (rangos) }\end{array}$ \\
\hline$n$ & 148 & 293 & 57 & 213 & 208 & 752 & 1.671 \\
\hline Neoplasia & 19 & $\underline{29}$ & 12 & 28 & 19 & $\underline{28}$ & $\underline{\mathbf{2 2 , 5}}(12-29)$ \\
\hline Bronquiectasias & 1 & 0,7 & $\underline{25}$ & 10 & $\underline{20}$ & 14,5 & $11,8(0,7-25)$ \\
\hline Bronquitis & $\underline{37}$ & 23 & 7 & $\underline{29}$ & 18 & 19,8 & $22,3(7-37)$ \\
\hline Tuberculosis & 5 & 3,1 & 16 & 3 & 0 & 1,7 & $5,8(1,7-5)$ \\
\hline Neumonía/abceso & 7 & 6,8 & 0 & 3 & 16 & 11,5 & $8,9(3-16)$ \\
\hline TEP & 1 & 0 & 0 & 0 & 0 & 2,3 & $1,6(1-2,3)$ \\
\hline Cardiopatía & 1 & 0,3 & 0 & 0 & 4 & 1,5 & $1,7(0,3-4)$ \\
\hline
\end{tabular}




\section{ESTUDIO INICIAL DEL PACIENTE CON HEMOPTISIS}

La inquietud que produce este síntoma tanto al paciente como al médico que lo atiende justifica que debamos tener claro cómo confirmar el origen del sangrado, valorar la gravedad del cuadro y la indicación de traslado del paciente a un centro hospitalario (Fig. 1) ${ }^{4}$, así como realizar una aproximación al diagnóstico etiológico y de localización del sangrado en el menor tiempo posible. Los pasos que debemos seguir ante todo paciente que acude con hemoptisis son los siguientes:

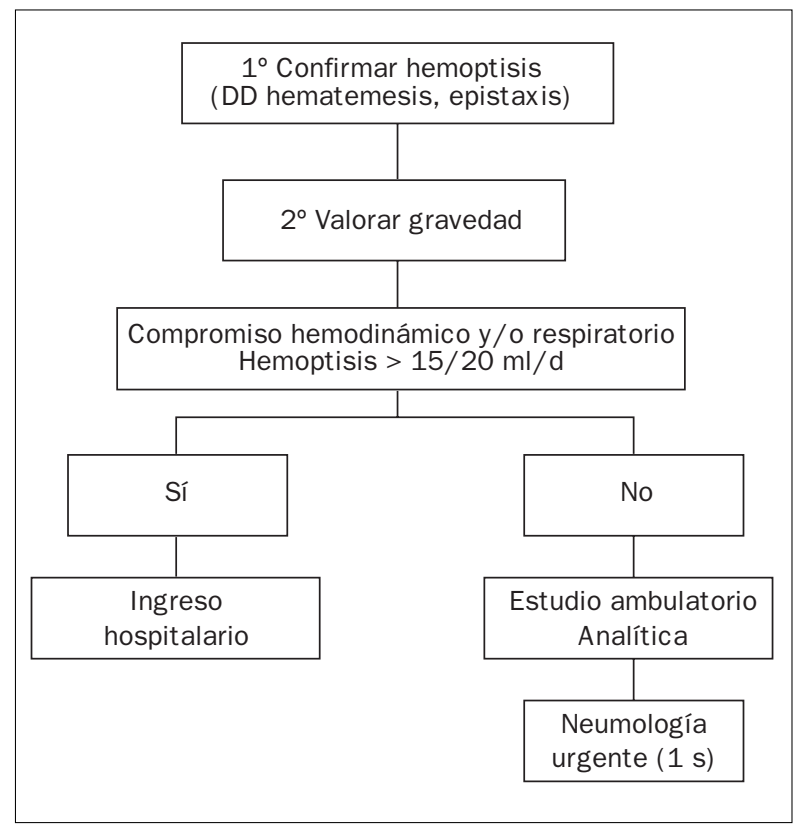

Figura 1

Manejo inicial de la hemoptisis4. DD: diagnóstico diferencial; $R x$ : radiografía; s: semana

\section{Confirmar la hemoptisis}

La sangre procedente del estómago o de la vía aérea superior se puede aspirar produciendo una irritación de la vía aérea subglótica dando lugar a la tos, de modo que es muy importante diferenciar la hemoptisis de la hematemesis y de la epistaxis a través de la clínica y la exploración física (Tabla III).

\section{Valoración de la gravedad}

Una vez confirmado que nos encontramos ante un paciente con hemoptisis, se valorará su gravedad. Ésta condicionará la urgencia con la que se debe realizar el estudio, estableciéndose dos grupos de trabajo: los que se deben derivar al hospital y aquéllos que se pueden estudiar de forma ambulatoria. El volumen de sangrado y la reserva funcional respiratoria son los factores pronósticos más importantes, tal y como muestra la figura 1 .

\section{Anamnesis}

Tranquilizado y estabilizado el paciente elaboraremos la historia clínica con el fin de orientar el estudio de la enfermedad responsable de la hemoptisis. En primer lugar recogeremos la edad, ya que tanto la estenosis mitral como la enfermedad bronquiectásica suelen observarse en pacientes de menos de 40 años, edad que generalmente sobrepasan los portadores de carcinoma pulmonar. Es importante conocer los antecedentes de enfermedades respiratorias, traumatismo torácico; posibilidad de aspiración de cuerpo extraño, así como episodios previos de hemoptisis. Investigaremos la toma de anticoagulantes y es muy importante conocer el hábito tabáquico del paciente tanto actual como en el pasado. Se anotarán los antecedentes de enfermedades sistémicas que puedan afectar al pulmón y que pueden cursar con hemoptisis (conectivopatías).

Con detalle se deben recoger las características del episodio actual de hemoptisis ${ }^{1}$, es decir, volumen total del sangrado en un tiempo determinado, tiempo de evolución y sintomatología acompañante, ya que nos permitirá realizar un diagnóstico provisional: la hemoptisis amenazante es más frecuente en pacientes con bronquiectasias, tuberculosis y carcinoma broncogénico; el esputo sanguinolento, sin material mucoide o purulento, asociado a dolor pleurítico y disnea súbita previa al sangrado es típico de infarto pulmonar; la presencia de sangre mezclada con pus junto a fiebre sugiere neumonía o abceso pulmonar, y bronquiectasias si el paciente presenta expectoración crónica abundante; ante sangre diluida, rosada y espumosa se debe sospechar edema agudo de pulmón; cambios en las características de la tos, junto con pérdida de peso y anorexia semanas antes de aparecer la hemoptisis hacen pensar en el carcinoma pulmonar.

\section{Exploración física}

Recordar que de manera inicial y periódicamente se debe realizar una valoración rápida del estado hemodinámico y del posible compromiso respiratorio $^{2}$. En la exploración física sistemática incluire$\operatorname{mos}^{7}$ : inspección de la cavidad oral y del área ORL; examen de la piel en busca de lesiones cutáneas como las telangiectasias (enfermedad de Rendu-Osler) o las chapetas malares (estenosis mitral); la exploración cardiaca puede poner de manifiesto signos de insuficiencia cardiaca congestiva o de valvulopatía mitral; la auscultación respiratoria podrá detectar crepitantes (neumonía, focos de hemorragia), roncus $y$ 
Tabla III

\begin{tabular}{|c|c|c|}
\hline \multicolumn{3}{|c|}{ DIAGNÓSTICO DIFERENCIAL ENTRE HEMOPTISIS Y HEMATEMESIS } \\
\hline & Hemoptisis & Hematemesis \\
\hline ¿Cómo expulsó la sangre? & Con la tos & Con el vómito \\
\hline ¿De qué color era? & Roja y espumosa & $\begin{array}{l}\text { Roja oscura o negra (posos de } \\
\text { café); nunca espumosa }\end{array}$ \\
\hline ¿De qué se acompañaba? & $\begin{array}{l}\text { Puede estar mezclada con } \\
\text { saliva }\end{array}$ & $\begin{array}{l}\text { Puede acompañarse de restos } \\
\text { alimenticios }\end{array}$ \\
\hline ¿Cuándo comenzó? & Hace varios días & De carácter agudo \\
\hline Otros síntomas & $\begin{array}{l}\text { Irritación faríngea, fiebre, } \\
\text { gorgoteo, tos, dolor costal, } \\
\text { expectoración purulenta }\end{array}$ & $\begin{array}{l}\text { Dolor abdominal, pirosis, } \\
\text { náuseas, vómitos }\end{array}$ \\
\hline Antecedentes personales & $\begin{array}{l}\text { EPOC, tuberculosis, } \\
\text { neoplasia pulmonar, }\end{array}$ & $\begin{array}{l}\text { Ingesta de gastroerosivos, ulcus, } \\
\text { hepatopatía }\end{array}$ \\
\hline Compromiso respiratorio & posible asfixia & Raro \\
\hline Reacción Labstix ${ }^{\circledast}(\mathrm{pH})$ & Alcalina & Ácida \\
\hline Anemia & Rara (hemoptisis masiva) & Casi constante \\
\hline Melenas & No & Habitualmente sí \\
\hline Confirmación & Broncoscopia & Endoscopia digestiva alta \\
\hline
\end{tabular}

EPOC: enfermedad pulmonar obstructiva crónica.

sibilantes diseminadoses (bronquitis crónica, bronquiectasias) o localizados (obstrucción bronquial por cuerpo extraño o tumoración).

\section{Radiografía de tórax}

La realización de una radiografía de tórax en proyección postero-anterior y lateral es imprescindible en el estudio inicial de la hemoptisis y un paso fundamental en el proceso diagnóstico, aunque la hemoptisis sea de grado leve ${ }^{5}$. En primer lugar, porque las causas del sangrado subglótico pueden dividirse en virtud de que la radiografía de tórax sea patológica o carezca de alteraciones significativas; y en segundo lugar, porque los hallazgos de la misma nos indicarán las sucesivas actuaciones diagnósticas y terapéuticas que debemos realizar.

Cuando aparecen alteraciones en la radiografia de tórax, se ha visto que las neoplasias son la causa más frecuente (Tabla IV). Se ha de tener en cuenta que las lesiones observadas pueden ser antiguas (valorarlo con radiografías previas) o bien corresponder a inundación del espacio aéreo por sangre de otro origen.

Si la radiografía de tórax es considerada normal o no localizadora (lo que ocurre en el $20-30 \%$ de los casos), las bronquitis y las bronquiectasias serán las causas más frecuentes en las que pensar. En estas circunstancias se observa una incidencia baja de carcinoma broncogénico de localización preferentemente central, que oscila según las series entre 1,7-11 ó 3-22\% de las hemoptisis idiopáticas ${ }^{1}$. Otras posibles causas de hemoptisis con radiografía de tórax sin alteraciones son las siguientes: falsa hemoptisis (patología bucal, epistaxis, hematemesis), alteración vascular pulmonar, diátesis hemorrágica, lesiones endobronquiales benignas (cuerpo extraño, adenoma bronquial, tuberculosis), insuficiencia cardiaca congestiva y hemosiderosis pulmonar idiopática.

\section{Estudio de laboratorio}

Es necesario realizar hemograma, bioquímica de rutina con perfil renal, sistemático de coagulación y estudio básico de orina con sedimento, para descartar una discrasia sanguínea así como para pensar en un síndrome pulmón-riñón. La sospecha clínica de conectivopatías nos obliga a solicitar un análisis más completo y específico. La gasometría arterial no es absolutamente necesaria, a no ser que exista un compromiso respiratorio previo o la situación global del paciente lo requiera.

Si el paciente sigue expectorando, se cursarán tres muestras seriadas de esputo para estudio microbiológico (baciloscopia y cultivo para micobacterias), y tres para el citológico, sobre todo en pacientes con factores de riesgo para neoplasia pulmonar ${ }^{1}$ (varón, mayor de 40 años y fumador), ya que sirven de gran interés en el seguimiento del paciente con hemorragia recidivante, radiografía de tórax normal y sin factores de riesgo para carcinoma broncogénico en el que no se cree necesario realizar pruebas invasoras (broncoscopia). 


\section{Tabla IV}

\begin{tabular}{|c|c|}
\hline \multicolumn{2}{|c|}{ CAUSAS DE HEMOPTISIS SEGÚN EL PATRÓN RADIOLÓGICO } \\
\hline \multirow[t]{4}{*}{ Atelectasia } & Tuberculosis pulmonar \\
\hline & Carcinoma de pulmón \\
\hline & Adenoma bronquial \\
\hline & Bronquiectasias \\
\hline \multirow[t]{3}{*}{ Patrón alveolar localizado } & Neumonía bacteriana \\
\hline & Tuberculosis pulmonar \\
\hline & Tromboembolismo pulmonar \\
\hline \multirow[t]{3}{*}{ Patrón alveolar difuso } & Neumonía \\
\hline & Tuberculosis pulmonar \\
\hline & Hemorragia alveolar \\
\hline \multirow[t]{7}{*}{ Cavitaciones } & Tuberculosis pulmonar \\
\hline & Carcinoma de pulmón \\
\hline & Abceso pulmonar \\
\hline & Neumonía cavitada \\
\hline & Bulla infectada \\
\hline & Cavidad con micetoma \\
\hline & Enfermedad de Wegener \\
\hline \multirow[t]{3}{*}{ Hilio patológico } & Carcinoma de pulmón \\
\hline & Tromboembolismo pulmonar \\
\hline & Tuberculosis pulmonar \\
\hline \multirow[t]{5}{*}{ Nódulos y masas } & Carcinoma de pulmón \\
\hline & Quiste hidatídico \\
\hline & Fístula arteriovenosa \\
\hline & Enfermedad de Wegener \\
\hline & Neumonía redonda \\
\hline \multirow[t]{4}{*}{ Derrame pleural } & Carcinoma de pulmón \\
\hline & Tromboembolismo pulmonar \\
\hline & Neumonía \\
\hline & Tuberculosis pulmonar \\
\hline
\end{tabular}

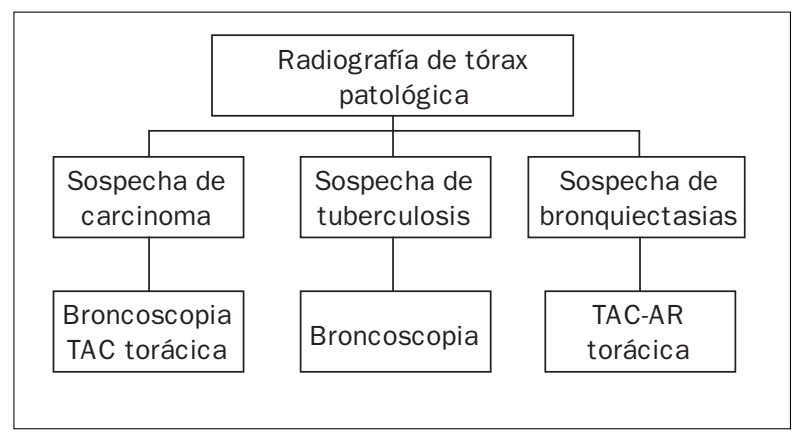

Figura 2

Pruebas complementarias en el estudio de la hemoptisis con Rx tórax localizadora de la lesión ${ }^{7}$; TAC: tomografía axial computerizada; TAC-AR: tomografía axial computerizada de alta resolución.

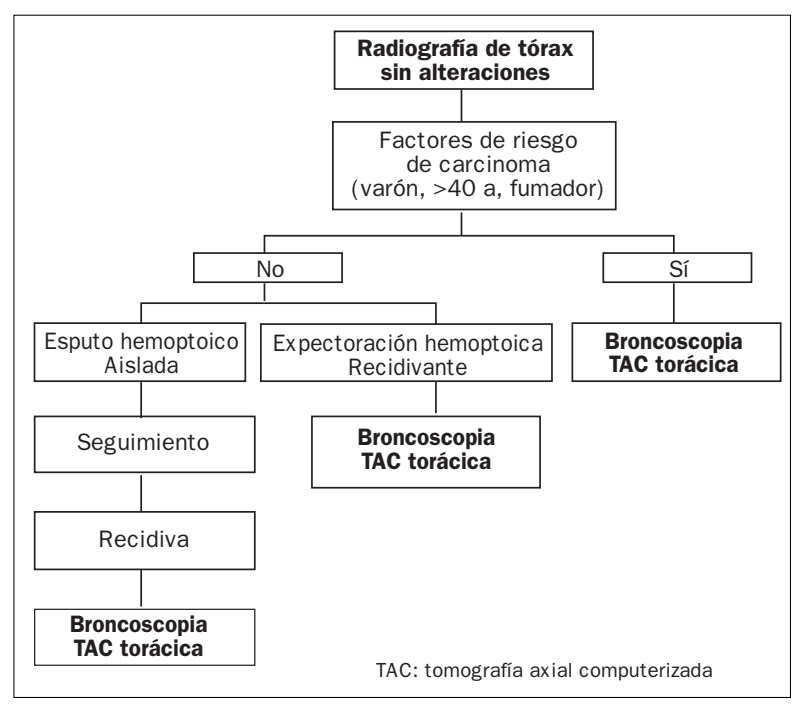

Figura 3

Pruebas complementarias en el estudio de la hemoptisis con Rx tórax no localizadora de la lesión ${ }^{7}$.

de zonas sospechosas y de muestras de zonas muy distales (no visualizadas) para estudio microbiológico y citológico, y el tratamiento de la pérdida hemática. Tras asegurar el estudio seriado de esputos y el estrecho control del paciente, puede prescindirse de la fibrobroncoscopia ante:

- Paciente menor de 40 años, no fumador, con episodio de esputo hemoptoico único sin otra sintomatología asociada y con una radiografía de tórax normal.

- Paciente con antecedentes clínicos importantes de una enfermedad no neoplásica que puede explicar la hemorragia (bronquiectasias).

- Paciente con hemorragia de origen extrapulmonar demostrado.

- Paciente con un estado clínico tan malo en el que no se llevaría a cabo ninguna acción independientemente de los hallazgos broncoscópicos. 
Por otra parte, en pacientes con radiografía de tórax patológica la rentabilidad diagnóstica de la broncoscopia es de hasta un $80 \%$, aumentando cuando se realiza de forma precoz (durante las primeras 24 horas), y disminuyendo a medida que aumenta dicho intervalo. Hasta en un tercio de los casos se encuentra un carcinoma broncogénico, pero hay que recordar que en pacientes con tumores periféricos la broncoscopia puede ser normal. Las situaciones que indicarían la fibrobroncoscopia aún en hemoptisis leve son:

- Paciente mayor de 40 años.

- Fumador de más de 40 paquetes/año.

- Hemoptisis durante más de 10 días.

- Clínica sugestiva de malignidad: astenia, anorexia, pérdida de peso, cambios en las características de la tos.

- Cambios radiológicos no justificados.

\section{TAC torácica}

La tomografía axial computerizada (TAC) torácica es una técnica de imagen muy útil en los pacientes con hemoptisis al revisar la vía aérea hasta la porción proximal de bronquios segmentarios, definir mejor el patrón radiológico, detectar lesiones no visibles en la radiografía, estudiar el mediastino y cuando se utiliza la TACAR (tomografía de alta resolución) es muy sensible en la detección de bronquiectasias.

\section{Otras exploraciones}

En función de la sospecha clínica, existen otras pruebas complementarias que nos ayudan al diagnóstico etiológico de la hemoptisis: la gammagrafía pulmonar en el tromboembolismo pulmonar, el ecocardiograma en la estenosis mitral, la gastroscopia si existen dudas sobre el origen del sangrado, la arteriografía pulmonar, etc.

\section{TRATAMIENTO INICIAL EXTRAHOSPITALARIO}

El manejo de estos pacientes puede iniciarse de forma ambulatoria por el médico de Atención Prima$r_{i a^{1,4}}$. En primer lugar se tranquilizará al paciente y a los familiares, y se tomarán medidas generales, como reposo relativo y antitusígenos (codeína $30 \mathrm{mg} / 6-8$ horas). El tratamiento específico dependerá de la sospecha diagnóstica, recomendando la abstención tabáquica a todos los fumadores. Se establecerá un estrecho control clínico, ya que aunque el paciente haya debutado inicialmente con un sangrado leve, puede presentar recidivas con hemoptisis más importantes que exijan una nueva evaluación ${ }^{6}$.

La mortalidad en pacientes con hemoptisis masiva se aproxima al $38 \%$, por lo que su manejo requiere una actuación inmediata para asegurar la permeabili- dad de la vía aérea y debe ser remitido urgentemente a un centro hospitalario adoptándose las siguientes medidas de soporte

1. Se contraindicará la ingesta de líquidos, alimentos o la toma de fármacos (dieta absoluta).

2. Se debe colocar al paciente en decúbito lateral sobre el pulmón supuestamente hemorrágico. Es poco frecuente que a través de la auscultación respiratoria se pueda determinar el lado sangrante, sin embargo, la detección de estertores o roncus en un área pulmonar específica puede ser de ayuda, máxime si coinciden con la existencia de lesiones radiológicas. A veces, es el propio paciente el que refiere notar de qué lado procede la sangre, refiriendo sensación de pesadez, gorgoteo o una molestia vaga en una parte del tórax. En caso de desconocer el origen de la hemoptisis es aconsejable adoptar la posición de Trendelemburg.

3. Se recomienda disponer de una vía venosa amplia, para perfundir suero salino fisiológico a una velocidad de perfusión que variará con relación al estado hemodinámico del paciente.

4. En sentido estricto sólo será necesario administrar oxígeno suplementario en caso de hipoxemia verificada por gasometría arterial. Sin embargo, como inicialmente no se dispone de ella, si existen signos físicos que sugieran compromiso respiratorio, se debe aplicar esta medida terapéutica de manera empírica.

5. Ante un paciente con hemoptisis y compromiso respiratorio, se le debe intubar preferentemente con tubo intratraqueal grueso $\left(\mathrm{n}^{\circ} 8\right)$ que permita la ventilación mediante ambú, y en caso de ser necesario, se aspirarán los coágulos de sangre. Si el paciente estuviera consciente, para intubarle se le relajará con midazolam $0,1 \mathrm{mg} / \mathrm{kg}$ vía venosa a ritmo lento.

\section{PRONÓSTICO Y SEGUIMIENTO}

Después de controlar y tratar el proceso de sangrado agudo, el pronóstico de la hemoptisis en general dependerá del pronóstico de la enfermedad causal así como del estado general del paciente ${ }^{2}$. De modo que la incertidumbre aparece en cuanto desconocemos la causa que ha originado el sangrado. ¿Volverá a repetirse el cuadro y con mayor intensidad? ¿Se trata de una enfermedad potencialmente grave que todavía no ha mostrado signos suficientes como para diagnosticarla? ¿Dónde, por quién, con qué pruebas y cada cuánto tiempo hay que vigilar a estos pacientes?

En primer lugar señalar que para poder definir la hemoptisis como de origen desconocido ${ }^{8}$ se precisa un examen endoscópico de las vías respiratorias subglóticas sin hallazgos patológicos que justifiquen el sangrado, además de la normalidad del resto de las pruebas diagnósticas comentadas (sistemático de coagulación, cultivo de esputo, radiografía de tórax, etc.). 
En general todos los autores afirman que el pronóstico de estos pacientes suele ser favorable, pero la inquietud generada por el antecedente de hemoptisis, sobre todo en pacientes de edad avanzada y fumadores, condiciona con frecuencia seguimientos prolongados. El pronóstico a corto plazo $^{8}$ de las hemoptisis criptogenéticas, cuando la fibrobroncoscopia es negativa, es habitualmente bueno. También se ha demostrado que el pronóstico a largo plazo $^{9}$ sigue siendo tan favorable como a corto plazo, que la recidiva no altera el pronóstico, y que no es necesaria la revisión prolongada de estos pacientes si no se presentan otros síntomas que alteren la evolución.

De modo que se confirma que el seguimiento en Atención Primaria debe realizarse sobre todo en los pacientes con factores de riesgo asociado $^{10}$, y en el Servicio de Neumología cuando la evolución clínica del paciente se pueda beneficiar de pruebas complementarias y/o de la valoración del especialista.

\section{CORRESPONDENCIA:}

Raquel Cortés Sancho

EAP Laguna de Duero

Avda. La Laguna, s/n

Laguna de Duero

Valladolid

\section{Bibliografía}

1. Jurado Gámez B, Capitán Narbona E, Ruiz Alcantarilla P. Expectoración hemoptoica: valoración en atención primaria. FMC 1996; 3, 2: 105-10.

2. Hirsberg B, Biran I, Glazer M, Kramer MR. Hemoptysis: etiology, evaluation, an outcome in a tertiary referral hospital. Chest 1997; 112:440-4.

3. Haro Estarriol M, Vicaya M, Jiménez J, Tornero A. Etiología de la hemoptisis: análisis prospectivo de 752 casos. Rev Clin Esp 2001; 201: 696-700.

4. Balcells Vilarnau E. Manejo de la hemoptisis en atención primaria. www.cap-semfyc.com; 2000:1-13.

5. Abal AT, Nair PC, Cherian J. Hemoptysis: etiology, evaluation and outcome-a prospective study in a third-world country. Respir Med 2001; 95: 548-52.
6. Haponik EF, Fein A, Chin R. Managing life-threatening hemoptysis. Has anything really changed? Chest 2000; 118: 1431-5.

7. Roig Cutillas J, et al. Normativa sobre el manejo de la hemoptisis amenazante. Arch Bronconeumol 1997; 33: 31-40.

8. Romero S, Martín C, Hernández L. Pronóstico a corto plazo de las hemoptisis criptogenéticas. Rev Clin Esp 1989; 185: 184-6.

9. Díaz Lobato S, Pino JM, Prados C, Villasante C. Pronóstico a largo plazo de las hemoptisis criptogenéticas. Rev Clin Esp 1990; 187: 99.

10. Herth F, Ernst A. Long-term outcome and lung cancer incidence in patients with hemoptysis of unknown origin. Chest 2001; 120: 1592-4. 